Indonesian Journal of Physics

Vol 19 No. 1, January 2008

\title{
Perturbative and Non-perturbative Aspects of the Chern-Simons-Witten Theory
}

\author{
Asep Yoyo Wardaya ${ }^{1,2, a)}$, F. P. Zen ${ }^{2,3, b)}$, Jusak Sali Kosasih ${ }^{2,3, c)}$, Triyanta $^{2,3, d)}$ \\ ${ }^{1)}$ Department of Physics, Diponegoro University, Semarang \\ ${ }^{2)}$ Indonesia Center for Theoretical and Mathematical Physics (ICTMP), \\ ${ }^{3)}$ Theoretical Physics Laboratory, THEPI Division, \\ Faculty of Mathematics and Natural Sciences, \\ Institut Teknologi Bandung, Bandung

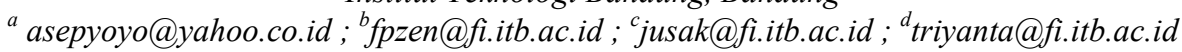

\begin{abstract}
We investigate a relation between non-perturbative and perturbative cases in the $2+1$ dimensional Chern-SimonsWitten (CSW) theory for $G=E_{6}$ gauge group. In the perturbative case, we calculate the vacuum expectation value (VEV) of an unknotted Wilson loop operator up to order $1 / k^{3}$ ( $k$ is a coupling constant). The result above is proved to be identical to the polynomial invariant $E_{0}(\rho)$ in the non-perturbative case at the same order of expansion.
\end{abstract}

Keywords: VEV, CSW, Wilson loop operator, non-perturbative, perturbative.

\section{Introduction}

The 2+1 dimensional Chern-Simons-Witten (CSW) theories have been extensively considered by mathematicians and physicists. In 1988, Edward Witten established the connection between ChernSimons gauge theory and the theory of knot and link invariants where there are the equivalence between vacuum expectation values (VEV) of Wilson loops (perturbative methods) and polynomial invariants (non-perturbative methods) $^{1,2)}$. In this paper we will show the equivalence explicitly for the group $G=E_{6}$.

We will restrict ourselves to the threedimensional manifold $\mathbb{R}^{3}$. Let $A$ be a $G$-connection. The usual CSW action is given by ${ }^{3,4)}$

$$
S_{C W W}=\frac{k}{4 \pi} \int_{R^{3}} d^{3} x \varepsilon^{\mu \nu \rho} \operatorname{Tr}\left(A_{\mu} \partial_{v} A_{\rho}+i \frac{2}{3} A_{\mu} A_{v} A_{\rho}\right),
$$

where $\operatorname{Tr}$ denotes the trace in the fundamental representation of $G$ and $k$ is a coupling constant. Summation over repeated indices is understood.

The Wilson loop operator $W_{\rho}$ is labeled by a

loop $\mathrm{C}$ embedded in $\mathbb{R}^{3}$ and a representation $\rho$ of $G$ and is defined as $^{3-6)}$

$$
W_{\rho}(C)=\operatorname{Tr}_{\rho}\left(P \exp \oint_{C} A\right) .
$$

In this equation, $P$ denotes path-ordered and $A=$ $A^{a} T_{a}^{(\rho)}$ with $T_{a}^{(\rho)}$ being the generator of $G$ in the representation $\rho$. The organization of the paper is the following. In Section 2 we will discussed the CSW theory by the use of non-perturbative method where the group $E_{6}$ is taken into consideration. Section 3, on the other hand, will discuss the same theory but perturbatively and compare the results with that from the previous section. The final section, Section 4, is devoted to conclusions.

\section{Non-Perturbative Methods in the CSW Theory} for Gauge Group $E_{6}$.

The polynomial invariant $E_{0}(\rho)$, given in the representation $\rho$, in the non-perturbative case of the CSW theory is taken into account in this section. For $E_{6}$ gauge group, $E_{0}(\rho)$ has been computed in Ref. 6.

In this section, we will discuss the computation of $E_{0}(\rho)$ by using the Braiding formula. From the decomposition of a tensor product $\rho_{a} \otimes \rho_{b}=\oplus_{t=1}^{r} \rho_{t}$, the following quadratic algebraic equation among $E_{0}(\rho)$ is fulfilled ${ }^{6-8)}$

$$
E_{0}\left(\rho_{a}\right) E_{0}\left(\rho_{b}\right)=\sum_{t=1}^{r} E_{0}\left(\rho_{t}\right) \cdot
$$

If $\rho_{a}$ and $\rho_{b}$ are the same representations, we can construct the equation

$$
q^{ \pm 2 Q\left(\rho_{a}\right)} E_{0}\left(\rho_{a}\right)=\sum_{t=1}^{r} \beta_{t} q^{ \pm \frac{1}{2} Q\left(\rho_{t}\right)} E_{0}\left(\rho_{t}\right),
$$

where the symmetry factor $\beta_{\mathrm{t}}$ equals $+1(-1)$ if $\rho_{t}$ is produced under (anti-) symmetry combination of the two $\rho_{a}$.

Now, we will apply the above expressions to the case of the $G=E_{6}$ group gauge. A decomposition of a tensor product of two fundamental representations 27 of $E_{6}$ is

$$
\underline{27} \otimes \underline{27}=\overline{351_{s}^{\prime}} \oplus \overline{351_{a}} \oplus \overline{27},
$$

where $s(a)$ denotes the index of (anti-) symmetric representation. The algebraic equations derived from it are

$$
\begin{aligned}
& E_{0}(27)^{2}=E_{0}\left(351^{\prime}\right)+E_{0}(351)+E_{0}(27), \\
& q^{ \pm 2 Q(27)} E_{0}(27)=q^{ \pm(1 / 2) Q\left(351^{\prime}\right)} E_{0}\left(351^{\prime}\right)
\end{aligned}
$$




$$
-q^{ \pm(1 / 2) Q(351)} E_{0}(351)+q^{ \pm(1 / 2) Q(27)} E_{0}(27) .
$$

Table 1. Quadratic Casimirs of the $E_{6}$ gauge group ${ }^{6)}$

\begin{tabular}{|c|c|c|c|c|}
\hline$Q(650)$ & $Q\left(351^{\prime}\right)$ & $Q(351)$ & $Q(78)$ & $Q(27)$ \\
\hline 18 & $\frac{56}{3}$ & $\frac{50}{3}$ & 12 & $\frac{26}{3}$ \\
\hline
\end{tabular}

Using the Quadratic Casimirs in table 1, we get a nontrivial solution:

$$
E_{0}(27)=[3]_{q^{2}}[9]_{\sqrt{q}},
$$

where notations in the eq. (8) are defined as

$$
[x]_{a}=\frac{a^{x}-a^{-x}}{a-a^{-1}}
$$

and

$$
q=\exp \left(\frac{2 \pi i}{k+Q(a d j)}\right) ; Q(A d j)=Q(78) .
$$

If eq. (8) is expanded in powers of $1 / \mathrm{k}$, we get

$$
E_{0}(27)=27-936 \frac{\pi^{2}}{k^{2}}+22464 \frac{\pi^{2}}{k^{3}}+\ldots .
$$

This is the polynomial invariant in the nonperturbative case of the CSW theory. This expression will be contrasted to the VEV of the Wilson loop operator in perturbative case of the same theory.

\section{Perturbative Methods of the Chern-Simons- Witten Theory}

In this section, we will do some computations in the CSW theory, perturbatively, i.e. we will calculate the VEV of the Wilson loop operator. Because of gauge invariance, in order to perform the quantization, we adopt the standard Faddeev-Popov procedure. Then, the total action is obtained by adding $S_{\text {gauge-fixing }}$ and $S_{\text {ghost }}$ to the usual CSW action ${ }^{3,4)}$ :

$$
\begin{gathered}
S_{\text {tot }}(A, \phi, c, \bar{c})=S_{C S W}(A)+S_{\text {gauge }- \text { fixing }}+S_{\text {ghost }} \\
=\frac{k}{4 \pi} \int_{M^{3}} d^{3} x \varepsilon^{\mu v \rho} \operatorname{Tr}\left(A_{\mu} \partial_{v} A_{\rho}+i \frac{2}{3} A_{\mu} A_{v} A_{\rho}\right) \\
+\frac{k}{4 \pi} \int_{M^{3}} d^{3} x \sqrt{g} g^{\mu v} A_{\mu}^{a} \partial_{v} \phi^{a} \\
-\int_{M^{3}} d^{3} x \sqrt{g} g^{\mu v} \partial_{\mu} \bar{c}^{a}\left(D_{\nu} c\right)^{a},
\end{gathered}
$$

where $\phi^{a}$ is the Lagrange multiplier (auxiliary field) and

$$
\left(D_{\mu} c\right)^{a}=\partial_{\mu} c^{a}-f^{a b c} A_{\mu}^{b} c^{c} .
$$

The action (11) gives rise gauge propagator and threegauge vertex. They are ${ }^{3,4)}$

$$
\begin{aligned}
\left\langle A_{\mu}^{a}(x) A_{v}^{b}(y)\right\rangle & =\frac{i}{k} \delta^{a b} \varepsilon_{\mu v \sigma} \frac{(x-y)^{\sigma}}{|x-y|^{3}}, \\
\left\langle A_{\mu}^{a}(x) A_{\nu}^{b}(y) A_{\rho}^{c}(z)\right\rangle & =-\frac{1}{4 \pi k^{2}} f^{a b c} \varepsilon^{\alpha \beta \gamma} \varepsilon_{\mu \alpha \sigma} \varepsilon_{\nu \beta \lambda} \varepsilon_{\rho \gamma \tau} \times
\end{aligned}
$$

$$
\times \int d^{3} w \frac{(w-x)^{\sigma}}{|w-x|^{3}} \frac{(w-y)^{\lambda}}{|w-y|^{3}} \frac{(w-z)^{\tau}}{|w-z|^{3}} .
$$

In the CSW theory one considers VEV of the Wilson loop operator defined as ${ }^{9)}$

$$
\left\langle W_{\rho}(C)\right\rangle=\frac{\int D A D \phi D c D \bar{c} \operatorname{Tr}_{\rho}\left(P \exp \oint_{c} A\right) \mathrm{e}^{i S_{t o t}}}{\int D A D \phi D c D \bar{c} \mathrm{e}^{i S_{t o t}}}
$$

In the eq. (14), the contribution of gauge-fixing and ghost fields vanish. Then, the VEV of the Wilson loop operator can be written in an expression in terms of propagators and vertices ${ }^{3,4)}$.

$$
\begin{aligned}
& \left\langle W_{\rho}(C)\right\rangle=\operatorname{Tr}_{\rho}\left[1+i \oint_{c} d x^{\mu}\left\langle A_{\mu}(x)\right\rangle\right. \\
& -\oint_{c} d x^{\mu} \int^{x} d y^{v}\left\langle A_{v}(y) A_{\mu}(x)\right\rangle \\
& -i \oint_{c} d x^{\mu} \int^{x} d y^{v} \int^{y} d z^{\sigma}\left\langle A_{\sigma}(z) A_{v}(y) A_{\mu}(x)\right\rangle \\
& +\oint_{c} d x^{\mu} \int^{x} d y^{v} \int^{y} d z^{\rho} \int^{z} d w^{\sigma}\left\langle A_{\sigma}(w) A_{\rho}(z) A_{v}(y) A_{\mu}(x)\right\rangle \\
& +i \oint_{c} d x^{\mu} \int^{x} d y^{v} \int^{y} d z^{\rho} \int^{z} d w^{\sigma} \int^{w} d v^{\lambda} \times \\
& \quad \times\left\langle A_{\lambda}(v) A_{\sigma}(w) A_{\rho}(z) A_{v}(y) A_{\mu}(x)\right\rangle \\
& -\oint_{c} d x^{\mu} \int^{x} d y^{v} \int^{y} d z^{\rho} \int^{z} d w^{\sigma} \int^{w} d v^{\lambda} \int^{v} d u^{\tau} \times \\
& \left.\times\left\langle A_{\tau}(u) A_{\lambda}(v) A_{\sigma}(w) A_{\rho}(z) A_{v}(y) A_{\mu}(x)\right\rangle+. .\right] .
\end{aligned}
$$

The VEV in eq. (15) can be expressed in powers of $(2 \pi / k)$. The term $(2 \pi / k)^{0}$ is contributed by $\operatorname{Tr}_{\rho}(1)=\operatorname{dim} \rho=27$. The $(2 \pi / k)$ term comes from

$$
\begin{aligned}
& -\operatorname{Tr}\left(R^{b} R^{a}\right) \oint_{c} d x^{\mu} \int^{x} d y^{v}\left\langle A_{v}^{b}(y) A_{\mu}^{a}(x)\right\rangle \\
& =-i\left(\frac{2 \pi}{k}\right) \operatorname{dim} \rho \cdot Q(27) \cdot \varphi(C),
\end{aligned}
$$

with the matrix form of the quadratic Casimir for the fundamental representation is given by

$$
Q(27) 1=R^{a} R^{a}
$$

and $\varphi(C)$ is equal to

$$
\begin{aligned}
& \varphi(C)=\frac{1}{4 \pi} \oint_{c} d x^{\mu} \oint_{c} d y^{v} \varepsilon_{\mu v \sigma} \frac{(x-y)^{\sigma}}{|x-y|^{3}} \\
& =\frac{1}{4 \pi} \int_{0}^{1} d s \int_{0}^{1} d t \varepsilon_{\mu v \sigma} \dot{x}^{\mu}(s) \dot{x}^{v}(t) \frac{(x(s)-x(t))^{\sigma}}{|x(s)-x(t)|^{3}} .
\end{aligned}
$$

Note that an explicit parameterization $\left\{x^{\mu}(t): 0 \leq t\right.$ $\leq 1$ \} of $C$ has been used in calculation of the eq. (18).

Let us analyze the $(2 \pi / k)^{2}$ part of $\left\langle W_{\rho}(C)\right\rangle$.

This can be written as

$$
\begin{aligned}
& \operatorname{Tr}_{\rho}\left[-i \oint_{c} d x^{\mu} \int^{x} d y^{v} \int^{y} d z^{\sigma}\left\langle A_{\sigma}(z) A_{v}(y) A_{\mu}(x)\right\rangle\right. \\
&\left.+\oint_{c} d x^{\mu} \int^{x} d y^{v} \int^{y} d z^{\rho} \int^{z} d w^{\sigma}\left\langle A_{\sigma}(w) A_{\rho}(z) A_{v}(y) A_{\mu}(x)\right\rangle\right]
\end{aligned}
$$




$$
\begin{aligned}
& =\left(\frac{2 \pi}{k}\right)^{2} \operatorname{dim} \rho \cdot Q(\operatorname{Adj}) \cdot Q(27) \cdot \varsigma_{1}(C) \\
& -\frac{1}{2}\left(\frac{2 \pi}{k}\right)^{2} \operatorname{dim} \rho \cdot Q(27)^{2} \cdot \varphi^{2}(C) \\
& \quad+\left(\frac{2 \pi}{k}\right)^{2} \operatorname{dim} \rho \cdot Q(\operatorname{Adj}) \cdot Q(27) \varsigma_{2}(C),
\end{aligned}
$$

where, for the $E_{6}$ gauge group, there are relations in quadratic Casimir : $\delta^{a b} Q(A d j)=f^{a c d} f^{b c d}$. Then, $\varsigma_{2}(C)$ comes from $\left\langle A_{\sigma}(w) A_{\rho}(z) A_{v}(y) A_{\mu}(x)\right\rangle$ term, that is

$$
\begin{gathered}
\varsigma_{2}(C)=\frac{1}{8 \pi^{2}} \oint_{C} d x^{\mu} \int^{x} d y^{v} \int^{y} d z^{\rho} \int^{z} d w^{\sigma} \times \\
\times \varepsilon_{\sigma v \alpha} \varepsilon_{\rho \mu \beta} \frac{(w-y)^{\sigma}}{|w-y|^{3}} \frac{(z-x)^{\sigma}}{|z-x|^{3}} .
\end{gathered}
$$

On the other hand, the contribution of the vertex $\left\langle A_{\sigma}(z) A_{v}(y) A_{\mu}(x)\right\rangle$ is

$$
\varsigma_{1}(C)=-\frac{1}{32 \pi^{3}} \oint_{C} d x^{\mu} \int^{x} d y^{y} \int^{y} d z^{\rho} H_{\mu v \rho}(x, y, z),
$$

with

$$
\begin{aligned}
& H_{\mu \nu \rho}(x, y, z)=\varepsilon^{\alpha \beta \gamma} \varepsilon_{\mu \alpha \sigma} \varepsilon_{\nu \beta \lambda} \varepsilon_{\rho \gamma \tau} \times \\
& \times \int d^{3} w \frac{(w-x)^{\sigma}}{|w-x|^{3}} \frac{(w-y)^{\lambda}}{|w-y|^{3}} \frac{(w-z)^{\tau}}{|w-z|^{3}} .
\end{aligned}
$$

We choose the unknot condition of the VEV above as the parameterization of an unit circle defined as

$$
U_{0}=\{x(s)=(\cos 2 \pi s, \sin 2 \pi s, 0): 0 \leq s \leq 1\} .
$$

Accordingly the values of $\varphi\left(U_{0}\right)$ and $\varsigma_{2}\left(U_{0}\right)$ vanish, while the value of $\varsigma_{1}\left(U_{0}\right)$ is $\left.3,4,10\right)$

$$
\begin{aligned}
& S_{1}\left(U_{0}\right)=-\int_{0}^{1} d s \int_{0}^{s} d t \int_{0}^{t} d u \dot{x}^{\mu}(s) \dot{x}^{v}(t) \dot{x}^{\rho}(u) \times \\
& \times\left(\frac{C_{1} C_{2} C_{3}}{32 \pi^{3}}\right)\left[\delta_{v \rho}(a-b)_{\mu}+\delta_{\mu \rho} b_{v}-\delta_{\mu \nu} a_{\rho}\right] \\
& =-\frac{1}{12}
\end{aligned}
$$

where

$$
\begin{gathered}
a=y-x=x(t)-x(s), \\
b=z-x=x(u)-x(s), \\
C_{1}(a, b)=\frac{2 \pi}{|a||b||a-b|}, \\
C_{2}(a, b)=\frac{1}{|a||b|+a_{\mu} b_{\mu}}, \\
C_{3}(a, b)=|a|+|b|-|a-b| .
\end{gathered}
$$

Now, we will compute $\left\langle W_{\rho}(O)\right\rangle$, the VEV of an unknotted Wilson loop operator at the order of $(2 \pi / k)^{2}$. For order $(2 \pi / k)^{0}$ and $(2 \pi / k)^{1}$, $\left\langle W_{\rho}(O)\right\rangle$ can be written as

$$
\begin{aligned}
\left\langle W_{\rho}(O)\right\rangle^{(1)} & =\operatorname{Tr}_{\rho}\left[1+i \Phi_{C} d x^{\mu}\left\langle A_{\mu}(x)\right\rangle\right] \\
& =27+0=27 .
\end{aligned}
$$

Furthermore, the $(2 \pi / k)^{2}$ parts of $\left\langle W_{\rho}(O)\right\rangle$ can be written as

$$
\begin{array}{r}
\left\langle W_{\rho}(O)\right\rangle^{(2)}=\left(\frac{2 \pi}{k}\right)^{2} \operatorname{dim} \rho \cdot Q(\operatorname{Adj}) \cdot Q(27) \cdot \varsigma_{1}\left(U_{0}\right) \\
=\left(\frac{2 \pi}{k}\right)^{2} 27.78 \cdot\left(\frac{26}{3}\right)\left(-\frac{1}{12}\right)=-936 \frac{\pi^{2}}{k^{2}} \cdot(27)
\end{array}
$$

Let us now continue our calculations to order $\left(\frac{2 \pi}{k}\right)^{3}$. The VEV of an unknotted Wilson loop operator in the $2+1$ dimensional Chern-Simons-Witten theory for this order is divided into two parts, that is $\left\langle W_{\rho}(C)\right\rangle^{(3 a)}$ and $\left\langle W_{\rho}(C)\right\rangle^{(3 b)} \cdot\left\langle W_{\rho}(C)\right\rangle^{(3 b)}$ involves $A^{6}$ term, combinations of three gauge propagators, of eq. (15), that is

$$
\begin{aligned}
-T_{\rho} & {\left[\oint_{c} d x^{\mu} \int^{x} d y^{v} \int^{y} d z^{\rho} \int^{z} d w^{\sigma} \int^{w} d v^{\lambda} \int^{v} d u^{\tau} \times\right.} \\
& \left.\times\left\langle A_{\tau}(u) A_{\lambda}(v) A_{\sigma}(w) A_{\rho}(z) A_{v}(y) A_{\mu}(x)\right\rangle\right] .
\end{aligned}
$$

This eq. (28) vanishes if the condition of parameterization (23) is taken into account:

$$
\left\langle W_{\rho}(O)\right\rangle^{(3 b)}=0 \text {. }
$$

The $\left\langle W_{\rho}(C)\right\rangle^{(3 a)}$, on the other hand, has the form of combinations of the propagator and the vertex ${ }^{11)}$

$\operatorname{Tr}_{\rho}\left[i \oint_{c} d x^{\mu} \int^{x} d y^{v} \int^{y} d z^{\rho} \int^{z} d w^{\sigma} \int^{w} d v^{\lambda} \times\right.$

$\left.\times\left\langle A_{\lambda}(v) A_{\sigma}(w) A_{\rho}(z) A_{v}(y) A_{\mu}(x)\right\rangle\right]$

$=\frac{i \cdot Q(\operatorname{Adj}) \cdot \operatorname{dim} \rho}{8 \pi k^{3}} Q(27)^{2} \oint_{c} d x^{\mu} \int^{x} d y^{v} \int^{y} d z^{\rho} \int^{z} d w^{\sigma} \int^{w} d v^{\lambda}$

$\times\left\{\varepsilon_{\lambda \sigma \alpha} \frac{(v-w)^{\alpha}}{|v-u|^{3}} H_{\rho v \mu}(y-z, x-z)+\varepsilon_{\lambda \rho \alpha} \frac{(v-z)^{\alpha}}{|v-z|^{3}} H_{\sigma \nu \mu}(y-w, x-w)\right.$

$+\varepsilon_{\lambda v \alpha} \frac{(v-y)^{\alpha}}{|v-y|^{3}} H_{\sigma p \mu}(z-w, x-w)+\varepsilon_{\lambda \mu \alpha} \frac{(v-x)^{\alpha}}{|v-x|^{3}} H_{\sigma p v}(z-w, y-w)$

$+\varepsilon_{\sigma \rho \alpha} \frac{(w-z)^{\alpha}}{|w-z|^{3}} H_{\lambda \nu \mu}(y-v, x-v)+\varepsilon_{\sigma v \alpha} \frac{(w-y)^{\alpha}}{|w-y|^{3}} H_{\lambda \rho \mu}(z-v, x-v)$

$+\varepsilon_{\sigma \mu \alpha} \frac{(w-x)^{\alpha}}{|w-x|^{3}} H_{\lambda \rho v}(z-v, y-v)+\varepsilon_{\rho v \alpha} \frac{(z-y)^{\alpha}}{|z-y|^{3}} H_{\lambda \sigma \mu}(w-v, x-v)$

$\left.+\varepsilon_{\rho \mu \alpha} \frac{(z-x)^{\alpha}}{|z-x|^{3}} H_{\lambda \sigma \nu}(w-v, y-v)+\varepsilon_{\nu \mu \alpha} \frac{(y-x)^{\alpha}}{|y-x|^{3}} H_{\lambda \sigma \rho}(w-v, z-v)\right\}$

$-\frac{i \cdot Q(A d j)^{2} \cdot \operatorname{dim} \rho}{16 \pi k^{3}} Q(27) \oint_{c} d x^{\mu} \int^{x} d y^{v} \int^{y} d z^{\rho} \int^{z} d w^{\sigma} \int^{w} d v^{\lambda} \times$ 


$$
\begin{aligned}
& \times\left\{\varepsilon_{\lambda \rho \alpha} \frac{(v-z)^{\alpha}}{|v-z|^{3}} H_{\sigma \nu \mu}(y-w, x-w)+\varepsilon_{\lambda v \alpha} \frac{(v-y)^{\alpha}}{|v-y|^{3}} H_{\sigma \rho \mu}(z-w, x-w)\right. \\
& +\varepsilon_{\sigma v \alpha} \frac{(w-y)^{\alpha}}{|w-y|^{3}} H_{\lambda \rho \mu}(z-v, x-v)+\varepsilon_{\sigma \mu \alpha} \frac{(w-x)^{\alpha}}{|w-x|^{3}} H_{\lambda \rho v}(z-v, y-v) \\
& \left.+\varepsilon_{\rho \mu \alpha \alpha} \frac{(z-x)^{\alpha}}{|z-x|^{3}} H_{\lambda \sigma v}(w-v, y-v)\right\} .
\end{aligned}
$$

Again, taking the unknot condition (23), terms in equation (30) have the values according to

$$
\begin{aligned}
& \oint_{c} d x^{\mu} \int^{x} d y^{v} \int^{y} d z^{\rho} \int^{z} d w^{\sigma} \int^{w} d v^{\lambda} \varepsilon_{\rho \mu \alpha} \frac{(z-x)^{\alpha}}{|z-x|^{3}} H_{\lambda \sigma v}(w-v, y-v) \\
& =32 i \pi\left(\frac{\pi^{2}}{6}-1\right) \text {, } \\
& \oint_{c} d x^{\mu} \int^{x} d y^{v} \int^{y} d z^{\rho} \int^{z} d w^{\sigma} \int^{w} d v^{\lambda} \varepsilon_{\sigma \mu \alpha} \frac{(w-x)^{\alpha}}{|w-x|^{3}} H_{\lambda \rho \nu}(z-v, y-v) \\
& =32 i \pi\left(\frac{\pi^{2}}{6}-1\right), \\
& \oint_{c} d x^{\mu} \int^{x} d y^{v} \int^{y} d z^{\rho} \int^{z} d w^{\sigma} \int^{w} d v^{\lambda} \times \\
& \times\left[\varepsilon_{\lambda \rho \alpha} \frac{(v-z)^{\alpha}}{|v-z|^{3}} H_{\sigma v \mu}(y-w, x-w)\right. \\
& \left.+\varepsilon_{\sigma v \alpha} \frac{(w-y)^{\alpha}}{|w-y|^{3}} H_{\lambda \rho \mu}(z-v, x-v)\right]=32 i \pi, \\
& \oint_{c} d x^{\mu} \int^{x} d y^{v} \int^{y} d z^{\rho} \int^{z} d w^{\sigma} \int^{w} d v^{\lambda} \varepsilon_{\lambda v \alpha} \frac{(v-y)^{\alpha}}{|v-y|^{3}} H_{\sigma \rho \mu}(z-w, x-w) \\
& =32 i \pi, \\
& \oint_{c} d x^{\mu} \int^{x} d y^{v} \int^{y} d z^{\rho} \int^{z} d w^{\sigma} \int^{w} d v^{\lambda} \varepsilon_{\lambda \mu \alpha} \frac{(v-x)^{\alpha}}{|v-x|^{3}} H_{\sigma \rho \nu}(z-w, y-w) \\
& =-32 i \frac{\pi^{3}}{6}, \\
& \oint_{c} d x^{\mu} \int^{x} d y^{v} \int^{y} d z^{\rho} \int^{z} d w^{\sigma} \int^{w} d v^{\lambda}\left\{\varepsilon_{\lambda \sigma \alpha} \frac{(v-w)^{\alpha}}{|v-w|^{3}} H_{\rho v \mu}(y-z, x-z)\right. \\
& +\varepsilon_{\sigma \rho \alpha} \frac{(w-z)^{\alpha}}{|w-z|^{3}} H_{\lambda v \mu}(y-v, x-v) \\
& +\varepsilon_{\rho v \alpha} \frac{(z-y)^{\alpha}}{|z-y|^{3}} H_{\lambda \sigma \mu}(w-v, x-v) \\
& \left.+\varepsilon_{v \mu \alpha} \frac{(y-x)^{\alpha}}{|y-x|^{3}} H_{\lambda \sigma \rho}(w-v, z-v)\right\}=-32 i \frac{\pi^{3}}{6} .
\end{aligned}
$$

Note that the calculations in equation (31.a-f) use the framing contour methods that have the solution

$$
\begin{aligned}
& x^{\mu}(t) \rightarrow y^{\mu}(t)=x^{\mu}(t)+\varepsilon n^{\mu}(t), \\
& n(s)=\left\{0,0, e^{\pi i s}\right\}, \\
& (\varepsilon>0,|n(t)|=1) .
\end{aligned}
$$

Therefore, for unknot condition (23), we get

$$
\begin{aligned}
\left\langle W_{\rho}(O)\right\rangle^{(3 a)} & =\frac{Q(A d j)^{2} \cdot \operatorname{dim} \rho \cdot Q(27)}{k^{3}} \cdot\left(\frac{2 \pi^{2}}{3}\right) \\
& =22464 \frac{\pi^{2}}{k^{3}}
\end{aligned}
$$

Finally, from the eq. (26), (27), (29) and (33) we obtained the VEV of the unknotted Wilson loop operator up to order $1 / k^{3}$, that is

$$
\left\langle W_{\rho}(O)\right\rangle=27-936 \frac{\pi^{2}}{k^{2}}+22464 \frac{\pi^{2}}{k^{3}}+\ldots . .
$$

It turns out that eq. (34) is identical to the eq. (10) in non-perturbative case.

\section{Conclusions}

We have obtained the explicit expression of the polynomial invariant of the CSW theory under the gauge group $E_{6}$, up to the third order in $(1 / k)$, by the used of the Braiding formula. We have also calculated explicitly the VEV of the unknotted Wilson loop operator in the same theory under the same gauge group, up to the third order in $(1 / k)$. The result is that both computations are identical, up to the same order. Since the polynomial invariant and the VEV of the Wilson loop operator describes the nonperturbative and perturbative aspects of the $2+1$ dimensional CSW theory, respectively, we conclude that the same results above show equivalence between both aspects in the $2+1$ dimensional CSW theory.

\section{Acknowledgements}

A.Y.W. would like to thank BPPS, Dirjen Dikti, Republic of Indonesia, for financial support. He also acknowledges all members of Theoretical Physics Laboratory, Department of Physics ITB, for warmest hospitality. This research is financially supported by Riset Internasional ITB No. 054/K 01.07/PL/2008.

\section{References}

1. E. Witten, Quantum Field Theory and The Jones Polynomial, LASSNS-HEP-88/33, Sept. 1988.

2. E. Witten, Gauge Theories and Integrable Lattice Models, Nucl. Phys. B., 322, 629-697, 1989.

3. E. Guadagnini, M. Martellini, M. Mintchev, Wilson Line in Chern-Simons Theory and Link Invariants, Cern-TH. 5420/89, IFUP-TH 24/89, May 1989.

4. E. Guadagnini, M. Martellini, and M. Mintchev, Chern-Simons Field Theory and Link Invariants, Cern-TH. 5479/89, Sept.1989.

5. M. Hayashi, F.P. Zen, Gravitational Scattering in $2+1$ Dimensions and Wilson Loop Operators, Progress of Theoretical Physics, 91:2, Februari 1994.

6. M. Hayashi, Calculation of Knot Polynomials for Unknotted Knot, Progress of Theoretical Physics, 90:1, July, 1993. 
7. F.P. Zen et al., Tetrahedron Diagram in ChernSimons-Witten Theory, Int. Adv. Tech. Congress CATS 2005, Kuala Lumpur, Malaysia, Dec. 2005.

8. F. P. Zen et al., HOMFLY Polynomial in SU(N) Chern-Simons-Witten Theory, Workshop on Theoretical Physics, Yogyakarta, 2006.

9. Dror Bar-Natan, Perturbative Chern-Simons Theory, J. Knot. Theor. Ramif., 26, 1995.
10. Triyanta et al., The Vacuum Expectation Values of Wilson Loop Operator in Chern-SimonsWitten Theory, Proc. Int. Conf. on Mathematics and Natural Sciences (ICMNS), Bandung, Nov. 29-30, 2006.

11. A. Y. Wardaya et al., Perturbative Calculation of the Chern-Simons- Witten Theory, Workshop on Theoretical Physics, Surabaya, 9 May 2007. 